\title{
Multiple Polymorphic Phases of A Mononuclear Spin-Crossover Fe(II) Complex with Significant Co-Ligand Effect
}

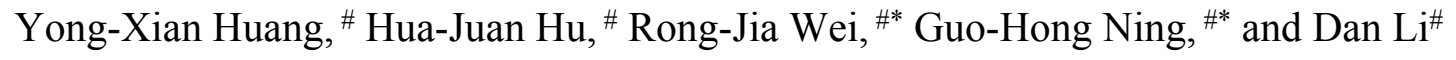

\#College of Chemistry and Materials Science, Guangdong Provincial Key Laboratory of Functional Supramolecular Coordination Materials and Applications, Jinan University, Huangpu Road West, Tianhe District, Guangzhou, Guangdong 510632, P. R. China

E-mail addresses: rjwei@jnu.edu.cn; guohongning@jnu.edu.cn 


\section{CONTENTS}

Section 1. General procedure

Section 2. Crystallographic data collection and refinement

Section 3. Additional crystallographic figures

Section 4. Magnetic properties

Section 5. IR spectra

Section 6. Solid-state UV-vis absorption spectra

Section 7. Hirshfeld surface Analysis.

Section 8. Reference 


\section{General procedure}

All starting materials were obtained commercially and were used without further purification. Elemental analyses for C, H, and N were performed on Perkin-Elmer 240Q elemental analyzer. The IR spectra (KBr pellets) were recorded in the range 400-4000 $\mathrm{cm}^{-1}$ on a Nicolet 5DX spectrophotometer. Powder X-ray diffraction (PXRD) studies were performed on Panalytical X-Pert PRO diffractometer with Mo K $\alpha$ radiation $(\lambda=$ $0.71073 \AA$ A). Magnetic susceptibility measurements were carried out on a Quantum Design MPMS XL7 magnetometer at a sweeping rate of $1 \mathrm{~K} \mathrm{~min}^{-1}$ in a cooling-heating cycle under magnetic field of 5000 Oe. Magnetic data were calibrated with the sample holder, and diamagnetic corrections were estimated from Pascal's constants. The Solidstate UV-vis absorption spectra were collected on a BioLogic MOS-500 circular dichroism spectrometer.

\section{Crystallographic data collection and refinement}

Single-crystal X-ray diffraction data for the polymorphs of $\left[\mathrm{Fe}(\mathrm{tpa})(\mathrm{NCSe})_{2}\right]$ were collected on Oxford Gemini S Ultra and Rigaku IP diffractometers with graphite monochromated radiation Mo $\mathrm{K} \alpha(\lambda=0.71073 \AA)$ source. Crystal structures were solved and refined using the SHELXTL program suite. ${ }^{1,2}$ Non-hydrogen atoms were generated by direct methods, which were refined first isotropically and then anisotropically. Hydrogen atoms were generated in calculated positions with the riding model. Crystal data and structure refinement parameters are summarized in Tables S1 and S2. The supplementary crystallographic data for SeI-IV at various temperatures can be obtained free at the CCDC database (2068657-2068664). 
Table S1. Crystal Data and Structure Refinements for SeI and SeII.

\begin{tabular}{|c|c|c|c|c|c|}
\hline & \multicolumn{2}{|c|}{ SeI } & \multicolumn{3}{|c|}{ SeII } \\
\hline formula & \multicolumn{2}{|c|}{$\mathrm{C}_{20} \mathrm{H}_{18} \mathrm{FeN}_{6} \mathrm{Se}_{2}$} & \multicolumn{3}{|c|}{$\mathrm{C}_{40} \mathrm{H}_{36} \mathrm{Fe}_{2} \mathrm{~N}_{12} \mathrm{Se}_{4}$} \\
\hline $\mathrm{fw} / \mathrm{g} \mathrm{mol}^{-1}$ & \multicolumn{2}{|c|}{556.17} & \multicolumn{3}{|c|}{1112.35} \\
\hline cryst syst & \multicolumn{2}{|c|}{ Monoclinic } & \multicolumn{3}{|c|}{ Monoclinic } \\
\hline space group & \multicolumn{2}{|c|}{$P 2_{1} / c$} & \multicolumn{3}{|c|}{$P 2_{1} / c$} \\
\hline$T / K$ & 298 & 150 & 300 & 230 & 150 \\
\hline$a / \AA$ & $12.8003(5)$ & $13.0230(8)$ & $22.2860(14)$ & 21.8701(11) & $22.3217(11)$ \\
\hline$b / \AA$ & $11.6476(5)$ & $11.3866(4)$ & $11.8329(5)$ & $11.7870(4)$ & $11.6957(5)$ \\
\hline$c / \AA$ & 17.6312(9) & 16.7181(14) & $17.3638(10)$ & $17.3045(8)$ & $16.9022(9)$ \\
\hline$a / \operatorname{deg}$ & 90 & 90 & 90 & 90 & 90 \\
\hline$b / \operatorname{deg}$ & 121.261(3) & $119.944(5)$ & $100.651(5)$ & $100.563(5)$ & $102.598(5)$ \\
\hline$r / \operatorname{deg}$ & 90 & 90 & 90 & 90 & 90 \\
\hline$V / \AA^{3}$ & 2247.03(17) & $2148.2(2)$ & $4500.1(4)$ & $4385.2(3)$ & $4306.4(4)$ \\
\hline$Z$ & 4 & 4 & 4 & 4 & 4 \\
\hline$D_{c} / \mathrm{g} \mathrm{cm}^{-3}$ & 1.644 & 1.720 & 1.642 & 1.685 & 1.716 \\
\hline$\mu / \mathrm{mm}^{-1}$ & 3.929 & 4.109 & 3.923 & 4.026 & 4.100 \\
\hline $\begin{array}{l}\text { reflns } \\
\text { collected }\end{array}$ & 18614 & 9069 & 15176 & 15979 & 17925 \\
\hline$R_{1}(I>2 s(I))^{a}$ & 0.0628 & 0.0491 & 0.0446 & 0.0421 & 0.0414 \\
\hline$w R_{2}$ (all data) & 0.131 & 0.0903 & 0.0914 & 0.0749 & 0.0716 \\
\hline
\end{tabular}


Table S2. Crystal Data and Structure Refinements for SeIII and SeIV.

\begin{tabular}{|c|c|c|c|}
\hline & & SeIII & SeIV \\
\hline formula & & $\mathrm{C}_{40} \mathrm{H}_{36} \mathrm{Fe}_{2} \mathrm{~N}_{12} \mathrm{Se}_{4}$ & $\mathrm{C}_{40} \mathrm{H}_{36} \mathrm{Fe}_{2} \mathrm{~N}_{12} \mathrm{Se}_{4}$ \\
\hline $\mathrm{fw} / \mathrm{g} \mathrm{mol}^{-1}$ & & 1112.35 & 1112.35 \\
\hline cryst syst & & Monoclinic & Triclinic \\
\hline space group & & $P 2_{1} / c$ & $P \overline{1}$ \\
\hline$T / K$ & 293 & 173 & 298 \\
\hline$a / \AA$ & $14.0458(8)$ & $13.8444(5)$ & $9.5345(4)$ \\
\hline$b / \AA$ & $21.7526(13)$ & $21.4130(8)$ & $15.6383(7)$ \\
\hline$c / \AA$ & $14.6855(10)$ & $14.5702(6)$ & $16.1336(7)$ \\
\hline$a / \operatorname{deg}$ & 90 & 90 & $74.1630(12)$ \\
\hline$b / \operatorname{deg}$ & $90.578(6)$ & $90.192(4)$ & $88.3460(12)$ \\
\hline$r / \operatorname{deg}$ & 90 & 90 & $75.7360(12)$ \\
\hline$V / \AA^{3}$ & $4486.7(5)$ & $4319.3(3)$ & $2241.02(17)$ \\
\hline$Z$ & 4 & 4 & 2 \\
\hline$D_{c} / \mathrm{g} \mathrm{cm}^{-3}$ & 1.647 & 1.711 & 1.648 \\
\hline$\mu / \mathrm{mm}^{-1}$ & 3.935 & 4.087 & 3.939 \\
\hline reflns collected & 30153 & 29940 & 8710 \\
\hline$R_{1}(I>2 s(I))^{a}$ & 0.0999 & 0.0681 & 0.0492 \\
\hline$w R_{2}$ (all data) & 0.1420 & 0.0952 & 0.1470 \\
\hline \multicolumn{4}{|c|}{${ }^{a} R_{1}=|| F_{\mathrm{o}}|-| F_{\mathrm{c}}|/| F_{\mathrm{o}} \mid ; w R_{2}=\left\{\left[w\left(F_{\mathrm{o}}^{2}-F_{\mathrm{c}}^{2}\right)^{2}\right] /\left[w\left(F_{\mathrm{o}}^{2}\right)^{2}\right]\right\}^{1 / 2}$} \\
\hline
\end{tabular}




\section{Additional crystallographic figures}

(a)

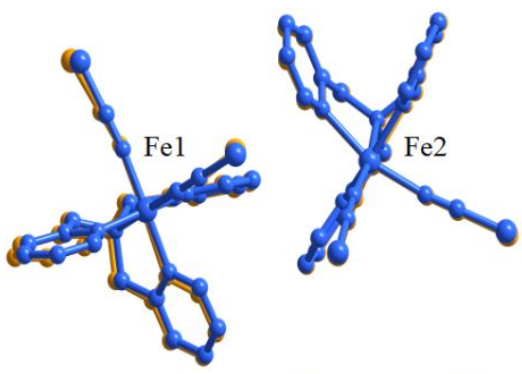

(b)

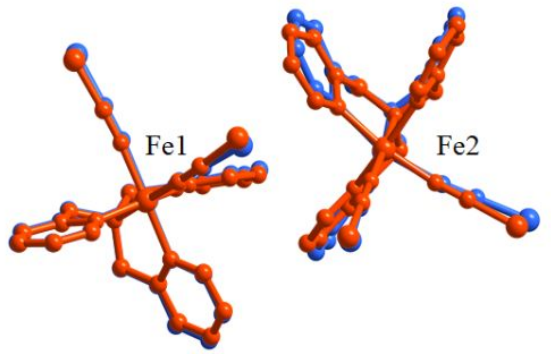

Figure S1. (a) Overlay of the molecular structures for SeII at 300 (yellow) and $230 \mathrm{~K}$ (blue). (b) Overlay of the molecular structures for SeII at 230 (blue) and $150 \mathrm{~K}$ (red).
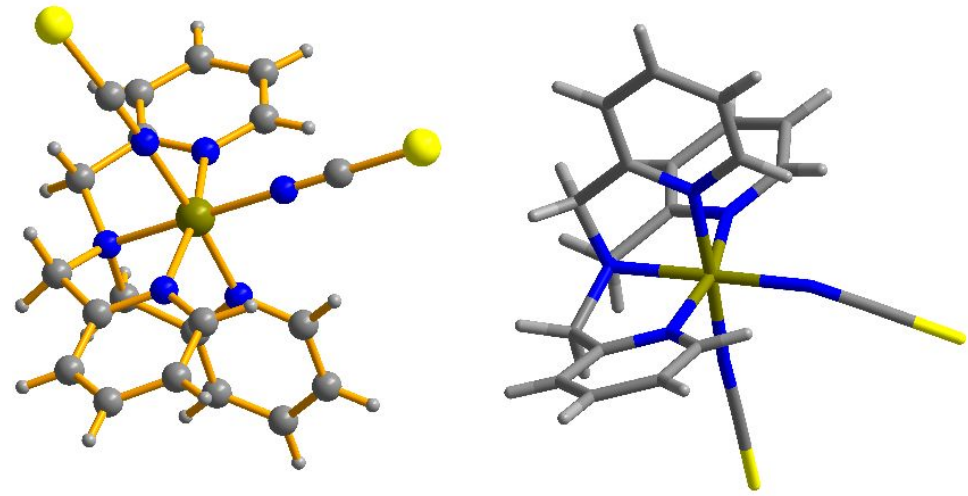

Figure S2. The asymmetric unit in SeIII. Fe1 units are shown in ball-and-stick form and $\mathrm{Fe} 2$ in capped-stick form. 


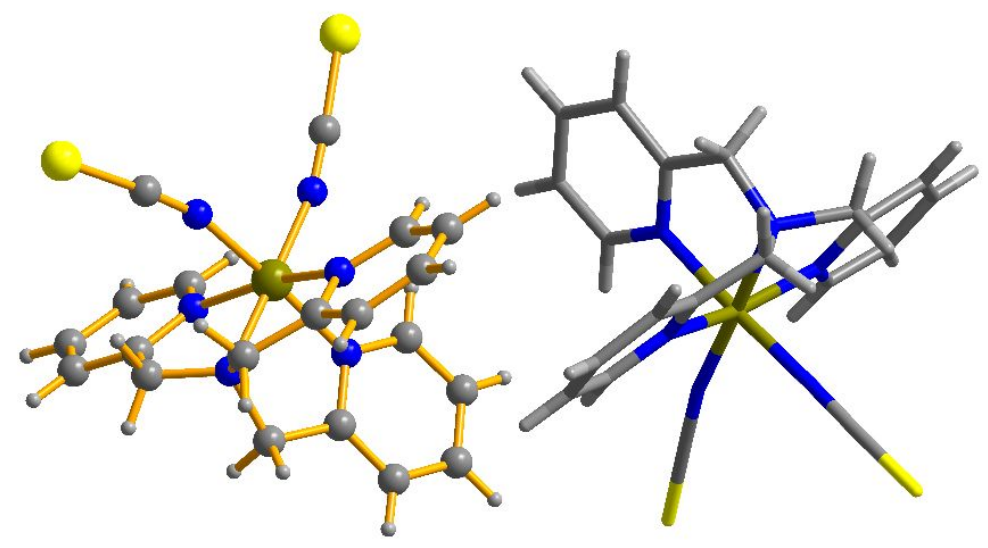

Figure S3. The asymmetric unit in SeIV. Fe1 units are shown in ball-and-stick form and $\mathrm{Fe} 2$ in capped-stick form.

\section{Magnetic properties}

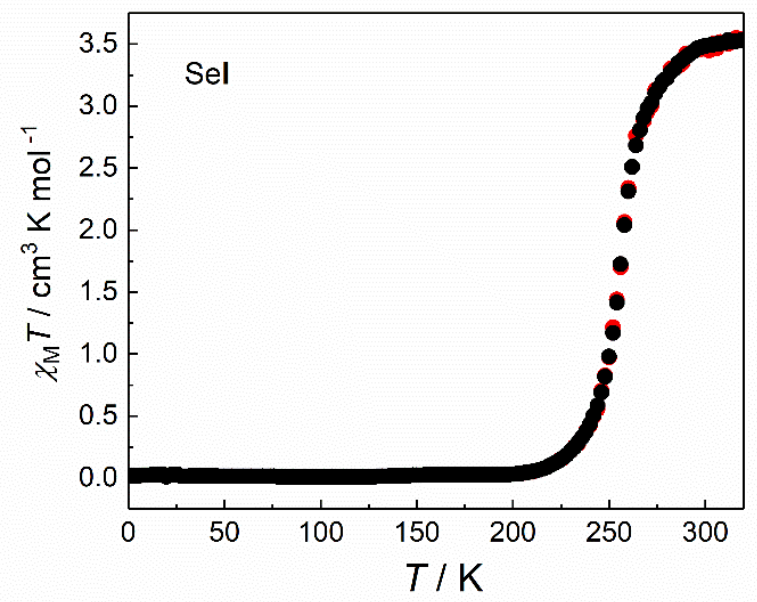

Figure S4. $\chi_{\mathrm{M}} T$ versus $T$ plots for SeI in a temperature cycles of $320-10-320 \mathrm{~K}$. 


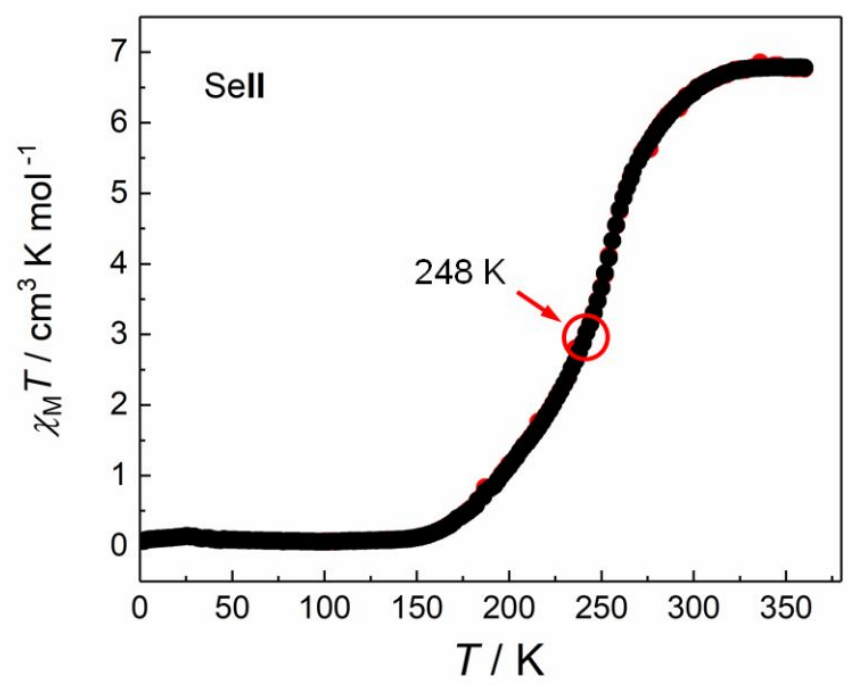

Figure S5. $\chi_{\mathrm{M}} T$ versus $T$ plots for SeII in a temperature cycles of $360-10-360 \mathrm{~K}$.

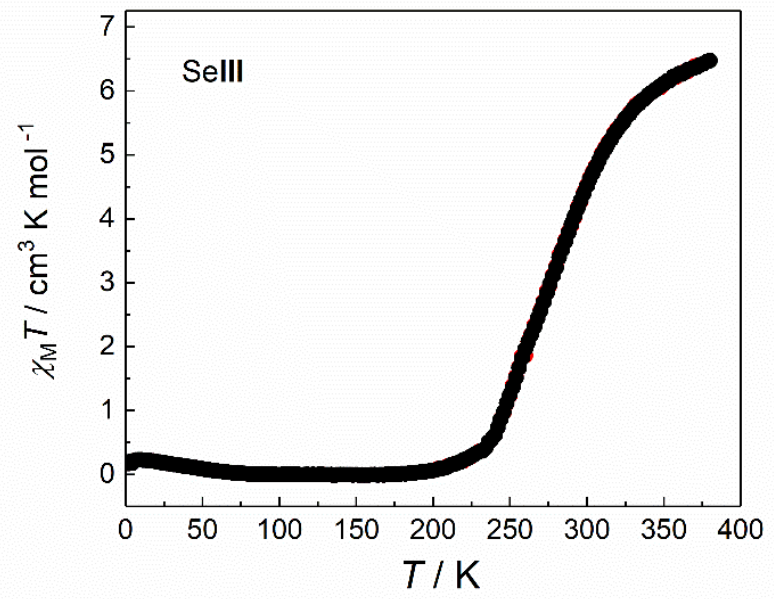

Figure S6. $\chi_{\mathrm{M}} T$ versus $T$ plots for SeIII in a temperature cycles of $380-10-380 \mathrm{~K}$.

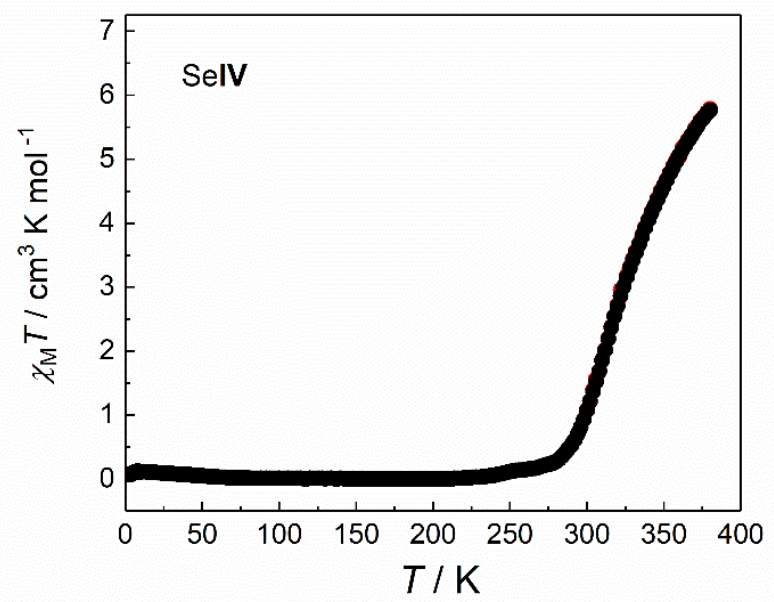

Figure S7. $\chi_{\mathrm{M}} T$ versus $T$ plots for SeIV in a temperature cycles of $380-10-380 \mathrm{~K}$. 


\section{IR spectra}

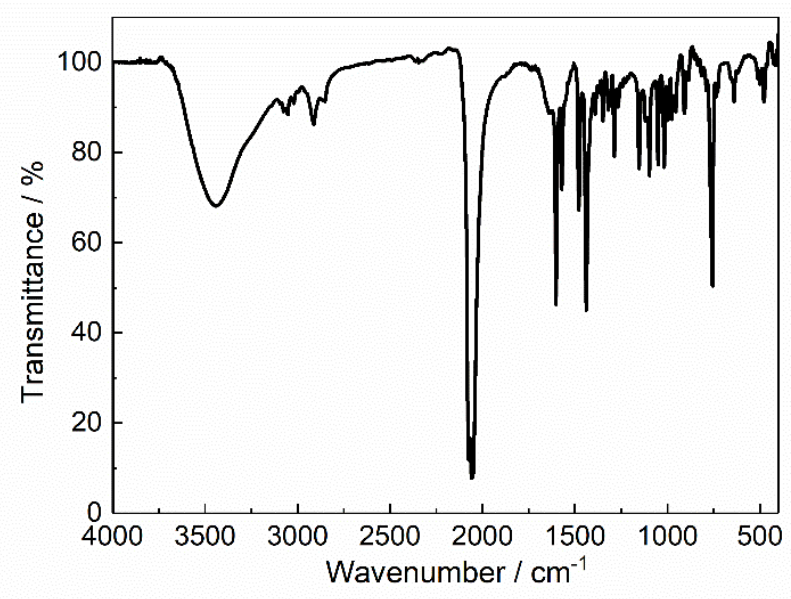

Figure S8. IR spectrum for SeI.

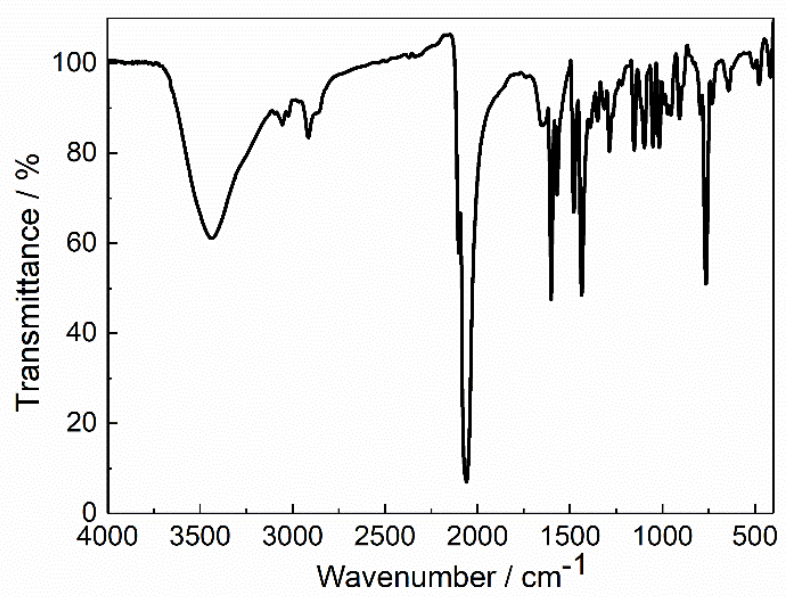

Figure S9. IR spectrum for SeII.

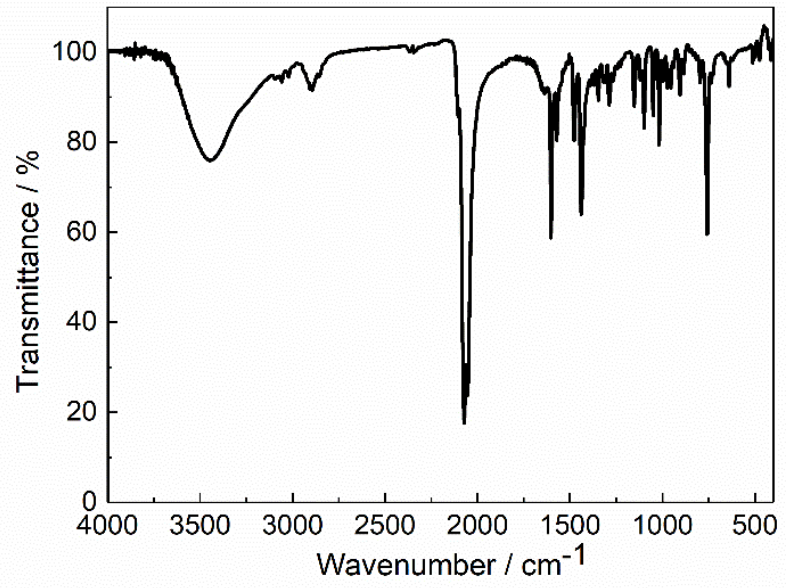

Figure S10. IR spectrum for SeIII. 


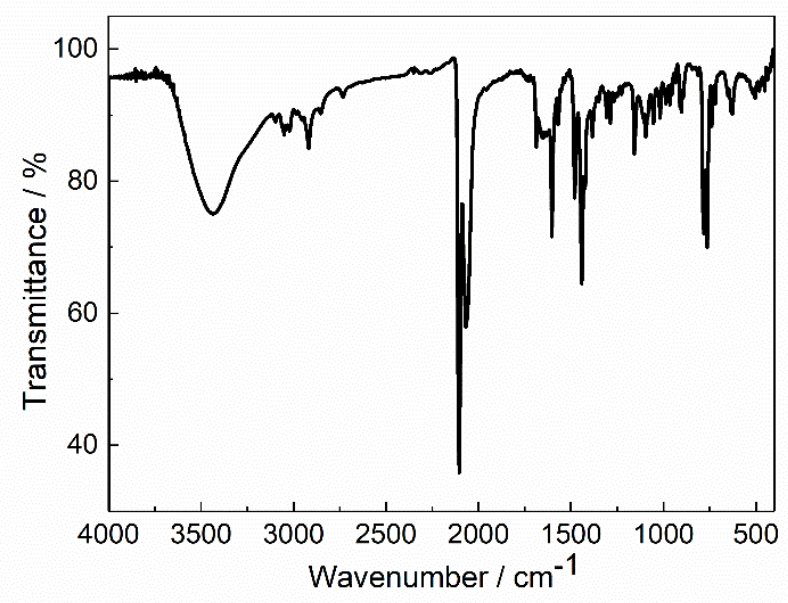

Figure S11. IR spectrum for SeIV.

\section{Solid-state UV-vis absorption spectra}

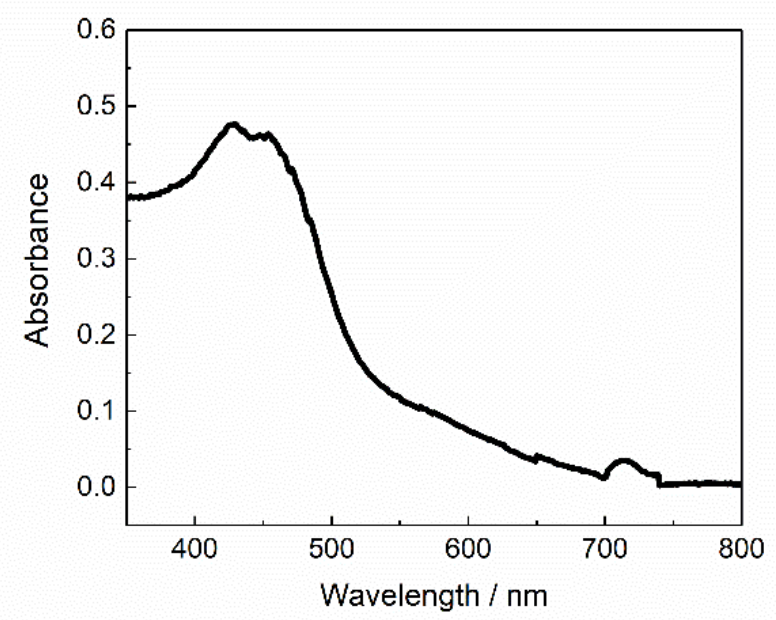

Figure S12. Solid-state UV-vis absorption spectrum for SeI. 


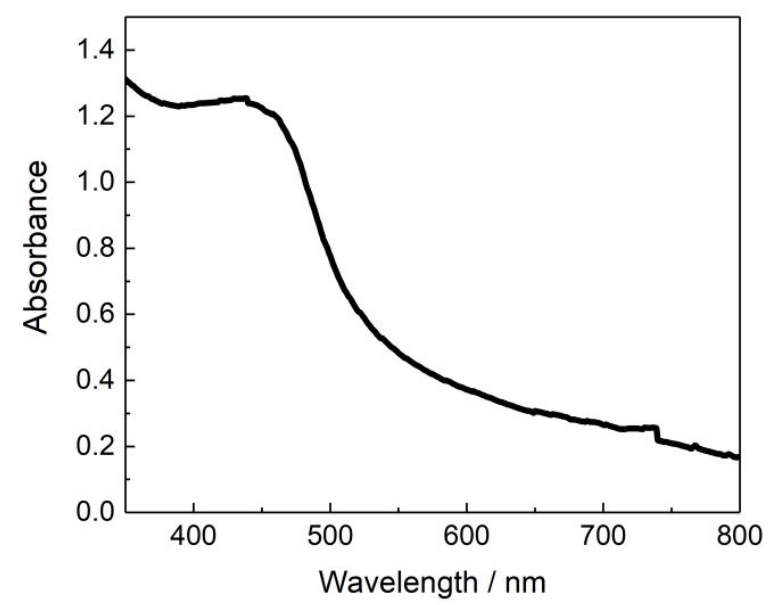

Figure S13. Solid-state UV-vis absorption spectrum for SeII.

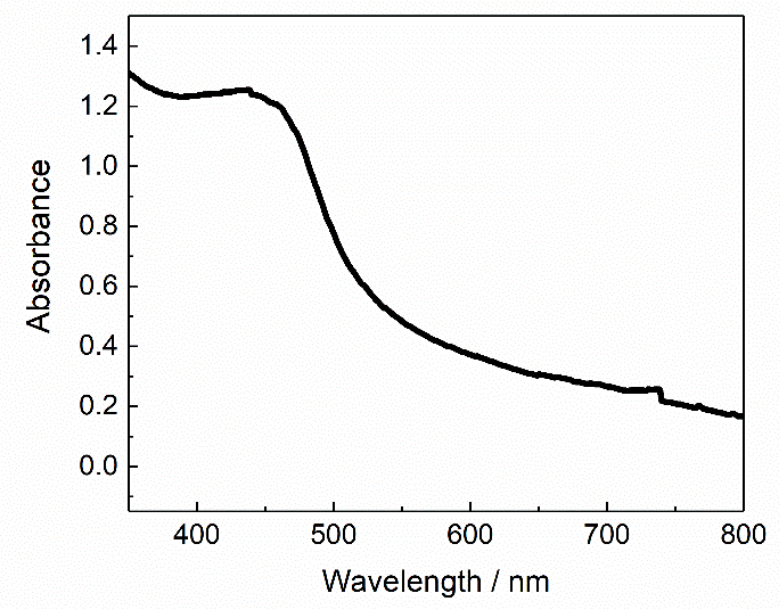

Figure S14. Solid-state UV-vis absorption spectrum for SeIII.

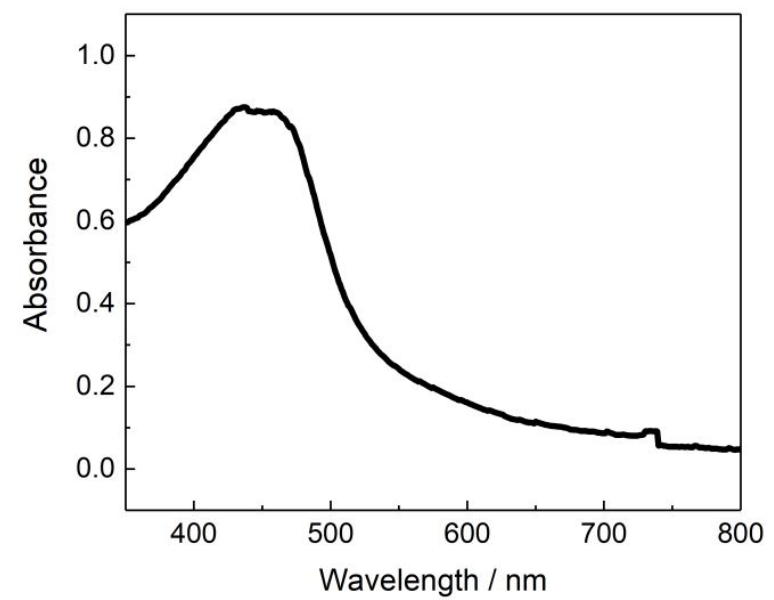

Figure S15. Solid-state UV-vis absorption spectrum for SeIV. 


\section{Hirshfeld surface Analysis}

Hirshfeld surface calculations ${ }^{\mathrm{S} 4}$ were performed by using the program CrystalExplorer 17.5. ${ }^{\mathrm{S} 5}$ Hirshfeld surfaces with $d_{\text {norm }}$ (isovalue $=0.5$ ) over the range -0.15 to 1.50 were mapped on each $\mathrm{NCS}^{-} / \mathrm{NCSe}^{-}$coligand for all complexes. $d_{\text {norm }}$ is the function of distance defined as follow:

$$
d_{n o r m}=\frac{d_{i}-r_{i}^{v d W}}{r_{i}^{v d W}}+\frac{d_{e}-r_{e}^{v d W}}{r_{e}^{v d W}}
$$

where $d_{\mathrm{i}}$ and $d_{\mathrm{e}}$ refer to the distances from a point on the surface to the nearest nucleus inside and outside the surface, respectively, while $r_{\mathrm{i}}^{\mathrm{vdW}}$ and $r_{\mathrm{e}}^{\mathrm{vdW}}$ refer to the vdW radii of the atoms. The red, white, as well as blue color regions in the surface mapped with $d_{\text {norm }}$ refer to the contact with the distance shorter than, equals to, and longer than sum of vdW radii, respectively. Each point on the Hirshfeld surfaces with $d_{\text {norm }}$ could be found on the corresponding 2D fingerprint with the coordinate of $\left(d_{\mathrm{i}}, d_{\mathrm{e}}\right)$.

(a)

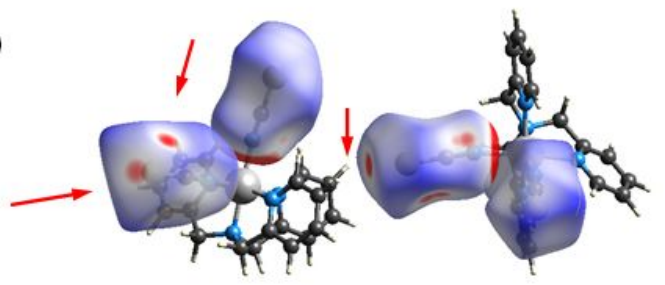

(b)

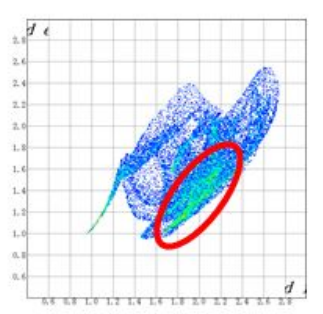

(d)

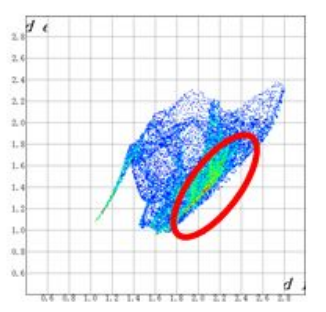

(c)

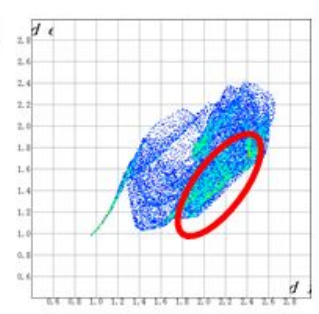

(e)

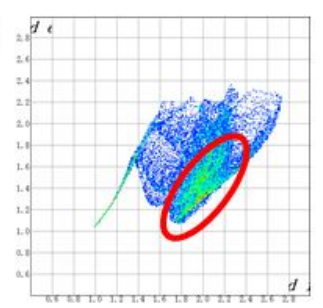

Figure S16. (a) Hirshfeld surfaces with dnorm (isovalue $=0.5$ ) over the range -0.15 to 1.50 (red through white to blue), showing notable intermolecular contacts in pII. The corresponding fingerprint plots for each $\mathrm{NCS}^{-}$group (from left to right) are shown in (b) to (e), respectively. The $\mathrm{S} \cdots \mathrm{H}$ hydrogen interactions are highlighted. 
(a)

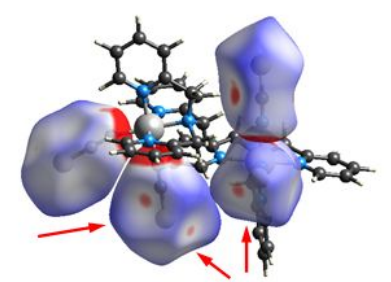

(b)

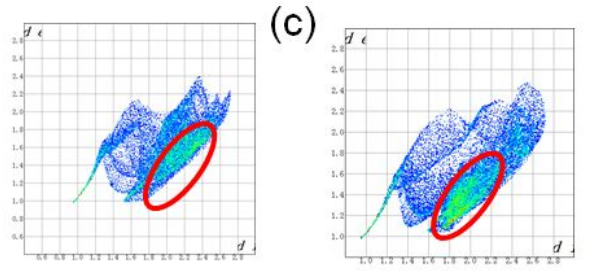

(d)

(e)

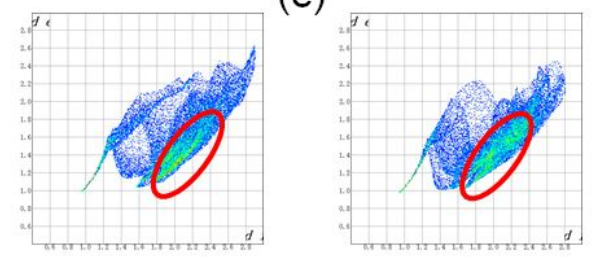

Figure S17. (a) Hirshfeld surfaces with dnorm (isovalue $=0.5$ ) over the range -0.15 to 1.50 (red through white to blue), showing notable intermolecular contacts in pIII. The corresponding fingerprint plots for each $\mathrm{NCS}^{-}$group (from left to right) are shown in (b) to (e), respectively. The $\mathrm{S} \cdots \mathrm{H}$ hydrogen interactions are highlighted.

(a)

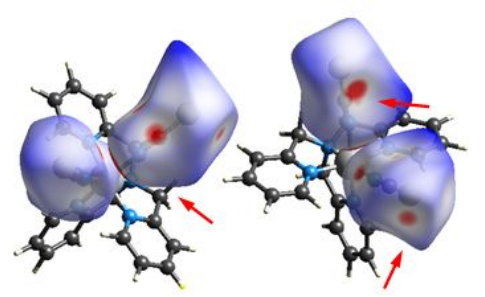

(b)

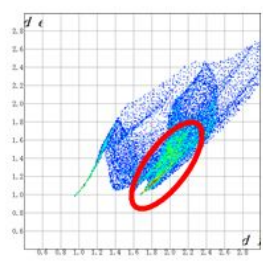

(d)

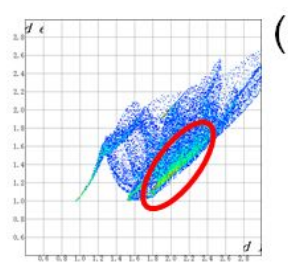

(c)

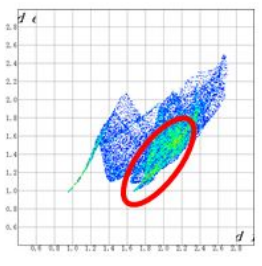

(e)

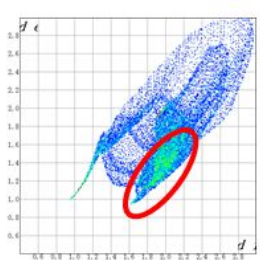

Figure S18. (a) Hirshfeld surfaces with dnorm (isovalue $=0.5$ ) over the range -0.15 to 1.50 (red through white to blue), showing notable intermolecular contacts in pIV. 
The corresponding fingerprint plots for each $\mathrm{NCS}^{-}$group (from left to right) are shown in (b) to (e), respectively. The $\mathrm{S} \cdots \mathrm{H}$ hydrogen interactions are highlighted.

(a)

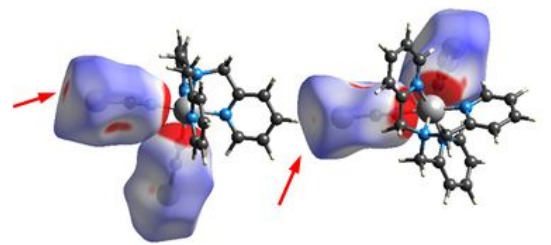

(b)

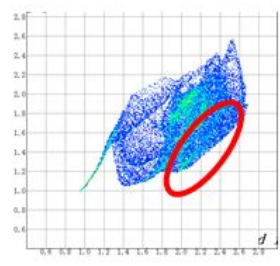

(d)

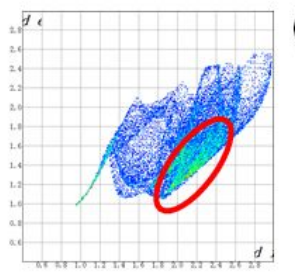

(c)

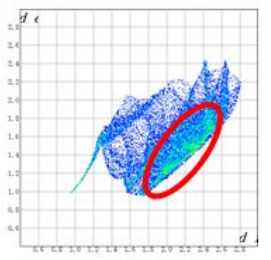

(e)

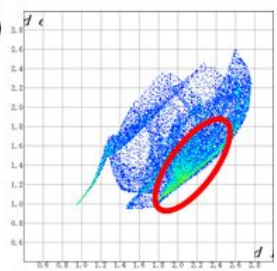

Figure S19. (a) Hirshfeld surfaces with dnorm (isovalue $=0.5$ ) over the range -0.15 to 1.50 (red through white to blue), showing notable intermolecular contacts in SeII. The corresponding fingerprint plots for each $\mathrm{NCSe}^{-}$group (from left to right) are shown in (b) to (e), respectively. The Se $\cdots H$ hydrogen interactions are highlighted.

(a)

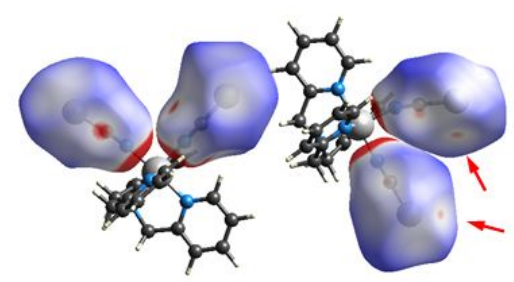

(b)

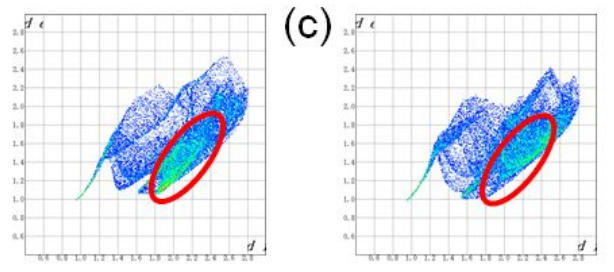

(d)

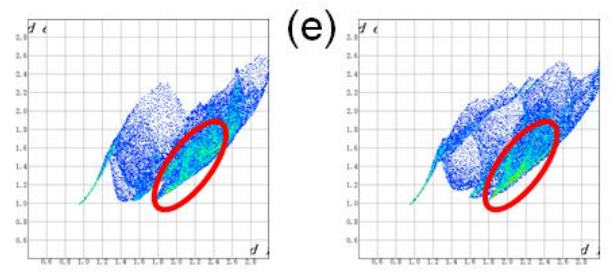


Figure S20. (a) Hirshfeld surfaces with dnorm (isovalue $=0.5$ ) over the range -0.15 to 1.50 (red through white to blue), showing notable intermolecular contacts in SeIII. The corresponding fingerprint plots for each $\mathrm{NCSe}^{-}$group (from left to right) are shown in (b) to (e), respectively. The Se $\cdots \mathrm{H}$ hydrogen interactions are highlighted.

(a)

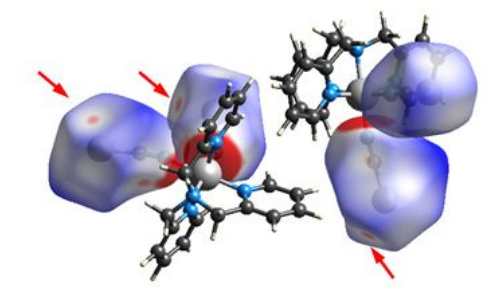

(b)

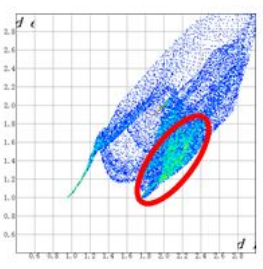

(d)

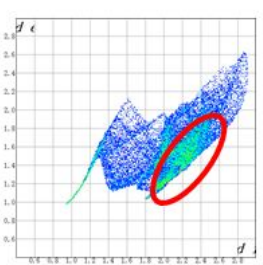

(c)

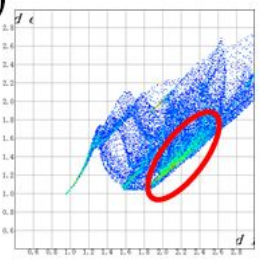

(e)

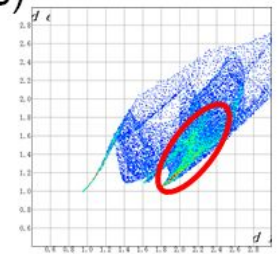

Figure S21. (a) Hirshfeld surfaces with dnorm (isovalue $=0.5$ ) over the range -0.15 to 1.50 (red through white to blue), showing notable intermolecular contacts in SeIV. The corresponding fingerprint plots for each $\mathrm{NCSe}^{-}$group (from left to right) are shown in (b) to (e), respectively. The Se $\cdots H$ hydrogen interactions are highlighted.

\section{Reference}

S1. Hübschle, C. B.; Sheldrick, G. M.; Dittrich, B. Shelxle: A qt graphical user interface for shelxl. J. Appl. Crystallogr. 2011, 44, 1281-1284.

S2. Sheldrick, G. M. A short history of shelx. Acta. Crystallogr. A. 2008, 64, 112-122.

S3. Spackman, M. A.; Jayatilaka. D. Hirshfeld surface analysis. CrystEngComm 2009, $11,19-32$.

S4. Turner, M. J.; McKinnon, J. J.; Wolff, S. K.; Grimwood, D. J.; Spackman, P. R.; Jayatilaka D.; Spackman, M. A. CrystalExplorer17 (2017). University of Western Australia. 